\title{
BMJ Open School-based interventions for preventing substance use in indigenous children ages 7-13: a scoping review protocol
}

\author{
Geoffrey Maina (D) , ${ }^{1}$ Taryn Phaneuf, ${ }^{2}$ Megan Kennedy, ${ }^{3}$ Maeve Mclean, ${ }^{4}$ \\ Ann Gakumo, ${ }^{5}$ Joseph Nguemo, ${ }^{6}$ Alexandra King, ${ }^{7}$ Solomon Kasha Mcharo ${ }^{8}$
}

To cite: Maina G, Phaneuf T, Kennedy M, et al. School-based interventions for preventing substance use in indigenous children ages 7-13: a scoping review protocol. BMJ Open 2020;10:e034032. doi:10.1136/ bmjopen-2019-034032

- Prepublication history and additional material for this paper are available online. To view these files, please visit the journal online (http://dx.doi. org/10.1136/bmjopen-2019034032).

Received 03 September 2019 Revised 22 January 2020 Accepted 22 January 2020

Check for updates

(C) Author(s) (or their employer(s)) 2020. Re-use permitted under CC BY-NC. No commercial re-use. See rights and permissions. Published by BMJ.

For numbered affiliations see end of article.

Correspondence to Dr Geoffrey Maina; geoffrey.maina@usask.ca

\section{ABSTRACT}

Introduction Throughout the world, indigenous peoples share traumatic colonial experiences that have caused gross inequalities for them and continue to impact every aspect of their lives. The effect of intergenerational trauma and other health disparities have been remarkable for Indigenous children and adolescents, who are at a greater risk of adverse mental health and addiction outcomes compared with non-indigenous people of the same age. Most indigenous children are exposed to addictive substances at an early age, which often leads to early initiation of substance use and is associated with subsequent physical and mental health issues, poor social and relational functioning, and occupational and legal problems. The aim of this paper is to report the protocol for the scoping review of school-based interventions for substance use prevention in Indigenous children ages 7-13 living in Canada, the USA, Australia and New Zealand. This scoping review seeks to answer the following questions: (1) What is known about indigenous school-based interventions for preventing substance use and (2) What are the characteristics and outcomes of school-based interventions for preventing substance use? Methods and analysis This scoping review will use steps described by Arksey and 0'Malley and Levac: (1) identifying the research question(s); (2) identifying relevant studies; (3) selecting the studies; (4) charting the data; (5) collating, summarising and reporting the results and (6) consulting with experts. Our findings will be reported according to the guidelines set by the Preferred Reporting Items for Systematic Reviews and Meta-Analyses extension for Scoping Reviews.

Ethics and dissemination Ethics review approval is not required for this project. Findings from this study will be presented to lay public, at scientific conferences and published in a peer-reviewed journal.

\section{BACKGROUND}

Indigenous peoples worldwide share traumatic colonial experiences that continue to impact every aspect of their lives. ${ }^{12}$ These historical sociopolitical processes have been attributed to causing gross inequalities for this population. ${ }^{3}$ Colonisation, the imposition of colonial institutions and rules,
Strengths and limitations of this study

- This scoping review will lead to a better understanding of the nature, characteristics and outcomes of interventions for indigenous preteen alcohol and substance use prevention.

- This study is limited to literature published in English between January 2009 and December 2019.

- Since the scoping review aims to assess the research activity on a topic and not the quality of the research thereof, this project will provide an overview of all the intervention focusing on elementary school-based indigenous substance use prevention.

- This study is also limited to research in Canada, the USA, Australia and New Zealand, and the findings will only be relevant to these countries.

and subsequent disruption of the aboriginal way of life continue to have significant negative impacts on the lives and health of indigenous people. In Canada, residential school experiences of physical, sexual and emotional violence, along with other legacies of colonisation, have had dramatic and far-reaching impacts that continue to cause negative health consequences for indigenous people. ${ }^{4}$ The resultant intergenerational trauma, which gets institutionalised within the family and the community, is associated with increased risks of mental health problems and addictions ${ }^{5-11}$

Colonisation is now regarded as a determinant of health, as it impacts many aspects of Indigenous peoples' well-being. ${ }^{12}$ Moreover, in Canada, aboriginal status is regarded as a determinant of health due to the numerous determinants of health they contend with, which are potentiated by colonial experiences. ${ }^{13-16}$ Thus, indigenous peoples lag the general population in social, economic and health indicators. ${ }^{17} 18$ Poverty, malnutrition, overcrowding and inadequate healthcare 
services continue to impact the health of the indigenous people. $^{19}$

Indigenous peoples carry a disproportionate burden of harms associated with substance use.$^{20}$ For example, compared with non-indigenous people who inject drugs, indigenous people who inject drugs are more likely to be infected with HIV and Hepatitis C and are less likely to receive treatment for substance use or have the support needed to remain on treatment. ${ }^{2122}$ The rates of opioid use and opioid use disorder, as well as associated mortality and morbidity, are higher among indigenous peoples compared with the general population. Furthermore, indigenous youth, young adults and young mothers are disproportionately affected by the opioid emergency. ${ }^{23-25}$

The sociopolitical factors that arose from colonial practices against indigenous people have also produced significant personal and structural barriers to health services. ${ }^{3}$ For example, indigenous people often experience racism in the healthcare system, limiting their access to health services. ${ }^{26-28}$

The effect of intergenerational trauma and other health disparities have been remarkable for indigenous children and adolescents, who are at a greater risk of adverse mental health outcomes. ${ }^{26}$ Suicide rates among Indigenous children and youth in Canada are 5-6 times higher than the national average. ${ }^{29-32}$ Among the Inuit youth, suicide rates are 11 times higher than the national average. Alcohol and substance use are risk factors for both committing and attempting suicide. ${ }^{33-35}$

About $20 \%$ of indigenous youth use substance use, of which $33 \%$ are under the age of 15 years. ${ }^{36}$ These early exposures frequently occur in children's homes and communities. For example, a Statistics Canada ${ }^{37}$ report on indigenous persons found that $35 \%$ of First Nation people living off-reserve, $30 \%$ of Metis and $39 \%$ of Inuit reported heavy drinking at least once a month for the past year, compared with $23 \%$ of non-indigenous people. Further, $34.5 \%$ of First Nations adults reported drinking alcohol two to three times a month, $17.9 \%$ drank two to three times a week and 3.2\% drank daily. ${ }^{23}$ Roughly twothirds $(63.6 \%)$ of First Nations adults who had consumed alcohol in the past 12 months meet the criteria for heavy drinking, with $16.0 \%$ reporting heavy drinking weekly. ${ }^{23}$

Early initiation of substance use is associated with development later in life of substance use-related mental health issues, poor social and relational functioning, and legal and occupational problems. ${ }^{38-40}$ Early onset of substance use may be a manifestation of an underlying vulnerability towards addictive behaviours or exposures to environmental conditions that increase or reinforce an individual's propensity towards substance use problems later in life. ${ }^{41-44}$ For instance, adolescents that use one class of substances, such as alcohol or tobacco, are more likely to progress to other illicit substances such as cannabis, methamphetamine or cocaine. ${ }^{38} 424546$ Disruption of brain development in adolescents using substances can increase risk of substance use disorders later in life. ${ }^{47}$ Early use of alcohol and substances can also complicate treatment outcomes for clients, who often manifest with physical and mental impacts of chronic polysubstance use. $^{18}$

Chen et $a t^{t 7}$ have established a link between the early onset of substance use and a greater propensity for substance use disorders later in life. Numerous studies suggest that the rate of substance use increases during adolescence, peaks in young adulthood and decreases in subsequent years. ${ }^{48}{ }^{49}$ Delaying the onset of substance use can, therefore, minimise the trajectory of substance use during adolescence and prevent the development of substance use disorders in adulthood. ${ }^{50} 51 \mathrm{~A}$ study authored by GM, an author in this manuscript on clients receiving methadone treatment for opiate use disorder, showed that clients were exposed to diverse substances at an early age alcohol 6 years, marijuana, 7 years, Dilaudid, morphine and cocaine (11 years), ritatin (8 years) and valium (13 years $\left.{ }^{22}\right]$. Studies show that indigenous children experience early exposure to substance use compared with non-indigenous people and are, likely to encounter diverse barriers, and determinants of health that may hinder access and retention to care. ${ }^{225253}$ Hence the need to focus on preventative measure for this population.

Elementary schools are a primary setting where preadolescent problems arise and where they can be prevented. School-based prevention programmes can target large numbers of preadolescents in a relatively cost-effective and efficient manner. ${ }^{54}$ Randomised control trials have been central to legitimising schoolbased substance use prevention programmes. ${ }^{55} 56$ Reviews of these trials have found that evidence-based programming in schools is effective in preventing the early onset of substance use and the escalation of substance use in adolescence. ${ }^{57-59}$

Effective school-based programmes often involve socialemotional learning competencies, cognitivebehavioural approaches, changes in school climate and multiyear and multicomponent approaches. ${ }^{60}$ Efforts such as these may delay or prevent the onset of substance use among preadolescents.

There is a critical need to understand how to design and implement effective school-based interventions for substance use prevention aimed at indigenous youth ages $7-13$. This scoping review aims to explore the research focusing on indigenous people living in developed countries, that is, Canada, the US, Australia and New Zealand. Although the authors recognise the distinct cultural and experiential differences between indigenous peoples of these countries, they share common historical experiences that impact their health. The authors intend to carry out interventions focusing on these populations in Canada. Therefore, limiting the literature search to these four countries will ensure that the results are relatable to the Canadian context. 


\section{METHODS}

The scoping review methodology has proven to be very useful for investigating the extent of the research in a given topic area. This type of synthesis review methodology was selected because of its rigorous and methodical approach that allows for openly framed research questions. Further, it was important that this review not be limited to only one evidence type (eg, only randomised controlled trials), as our preliminary search determined that the literature included a variety of qualitative evidence sources.

To ensure accurate and thorough reporting, the scoping review will be conducted following the six-stage approach described by Arksey and O'Malley ${ }^{61}$ and refined by Levac et $a l^{621}$ : identifying the research question(s), ${ }^{2}$ identifying relevant studies, ${ }^{3}$ selecting the studies, ${ }^{4}$ charting the data, ${ }^{5}$ collating, summarising and reporting the results, and $^{6}$ consulting with experts. Further, the review will be reported using guidelines from the Preferred Reporting Items for Systematic Reviews and Meta-Analyses extension for Scoping Reviews (PRISMA-ScR).

\section{Ethics and dissemination}

Findings from this study will be presented to lay public, at scientific conferences and published in a peer-reviewed journal.

\section{Stage 1: identifying the research questions}

To start the process, we assembled a team of scholars with experiences working with indigenous and marginalised communities. Consultations with the Health Science librarian helped her identify key phrases and words that represented the broader focus of Indigenous Elementary Substance use prevention. The research team identified one broad research question: (1) what is known about indigenous elementary school-based interventions for preventing substance use? We chose to focus on this population because GM's research indicating that clients with addictions had been exposed to substances at a very young age. ${ }^{22}$ The librarian (MK) ran a trial search to determine the feasibility of this research question. Following Arksey and O'Malley, ${ }^{61}$ our research question was refined as we became more familiar with the literature. Aiming to carefully examine and map the evidence on school-based interventions for preventing substance use in indigenous children ages 7-13, we developed the following research questions:

1. What is known about elementary indigenous schoolbased interventions for preventing substance use?

2. What are the characteristics and outcomes of elementary school-based interventions for preventing substance use?

\section{Stage 2: identifying relevant studies}

As scoping reviews allow for flexibility in evidence type, our review will not be limited to any specific qualitative or quantitative study design. To efficiently conduct searches in electronic databases, some parameters are needed to help guide the search strategy.

\section{Eligibility criteria}

The following inclusion criteria were identified and used to guide the searches and review the articles: (1) Only English language articles published between 2009 and 2019 will be included. Limiting the project to the last 10 years is informed by the authors' belief that due to ever-changing and dynamic substance use and addiction landscape, interventions older than 10 years might not be clinically relevant for informing interventions for elementary school children. (2) The population targeted in the studies that identify Indigenous children ages 7-13 living in North America (Canada or USA), Australia or New Zealand as one of the target population for intervention; (3) Studies that comment on mixed-age populations of indigenous children and adolescents will be included for full-text analysis to further explore their suitability for inclusion and (4) Studies will be discussing or presenting the intervention or the results of interventions for schoolbased substance use prevention for this target population.

\section{Exclusion criteria}

The following exclusion criteria were identified: (1) articles discussing interventions for addictions such as smoking, gambling, internet/social media/technology, (2) articles discussing adult population (ages 18+) and (3) review articles and commentaries.

\section{Database searches}

With input and in consultation with the research team, MK, an experienced health sciences librarian and familiar with evidence synthesis and reporting, established and tested the search strategy in MEDLINE identified relevant keywords. Controlled vocabulary was used to enhance sensitivity and specificity within the search. This preliminary search was developed and run to determine the feasibility of the scoping review and inform the searches in additional databases.

The following electronic databases will be searched for published literature: MEDLINE in-process and other nonindexed citations (Ovid) -1946 to present; PubMed-1966 to present; EMBASE Classic (Ovid) 1947 to present; Cumulative Index of Nursing and Allied Health Literature (CINAHL) (EBSCO) - 1937 to present; Educational Resources Information Centre (Ovid) -1965 to present; Scopus (Elsevier) -1970 to present and Cochrane Library (Wiley) and PsycINFO (1806 to present).

The results from each database search will be documented and saved, and references will be imported into EndNote, a bibliographic management software. Following the removal of duplicate references from EndNote, references will be imported into Rayyan, a review software ${ }^{63}$ for title and abstract screening. Google Forms will be used to collect data during full-text analysis.

To facilitate retrieval of relevant articles, the search was limited by language (English) and publication date (2009-2019). See online supplementary appendix A for full MEDLINE search strategies. In addition to searching 
electronic databases, the Canadian Agency for Drug and Technology in Health $(\mathrm{CADTH})^{64}$ tool will be used to search for relevant grey literature. The relevant areas to be searched from the $\mathrm{CADTH}^{64}$ tool will include Health Technology Assessment agencies (Canada, Australia and USA), free and subscription-based databases, internet search engines, mental health and nursing. Keywords identified throughout our search process will be used in the search process. We will also check reference lists of included studies to identify any that had not been found in our other searches.

\section{Stage 3: study selection}

Rayyan will be used for the title/abstract screening process. The research team will identify inclusion/exclusion keywords in correlation with identified inclusion and exclusion criteria. For example, exclusion keywords for smoking, gambling as identified in exclusion criterion 1. Two reviewers will independently review all articles. Should these 2 reviewers not reach a consensus, a third reviewer will complete the title and abstract screening. This process will be repeated during the full-text analysis; however, the mechanism for recording data and reviewing full-text articles will be by EndNote. Google survey will be used to collect data (see below, 'Charting the data').

\section{Stage 4: charting the data}

Review data extracted into a Google survey will include (1) author(s), title, (2) age groups of the target population, (3) indigenous population identified, including the country of residence, (4) summary of intervention characteristics and (5) intervention outcomes (yes/no).

\section{Stage 5: collating, summarising and reporting the results}

The purpose of a scoping review is to map and aggregate findings to present an overview of the topic. Accordingly, we plan to (1) map the results (main sources, locations and quantity), (2) provide a descriptive summary and qualitative analysis, (3) identify conceptual definitions, (4) provide a glossary of terms to clarify definitions found in the literature and (5) report our results using the PRISMA-ScR guidelines for scoping reviews to enhance transparency and reproducibility. Because this is a scoping review, we will not appraise the quality of the studies or offer statistical analysis.

Information to be extracted and compiled into a table include; title of the articles, country of focus, substance use intervention, intervention type, main findings and lessons that can be learnt. Thematic analysis will be applied to identify common threads that will emerge from the data.

\section{Stage 6: consulting}

We will present the preliminary findings of this scoping review to an advisory group comprised of an Indigenous elder, a knowledge keeper, elementary school leadership and community members of one of the communities GM has a collaborative relationship with and one whose children are impacted by substance use. Community partners working with GM will help to identify the appropriate advisory team members to join the committee. Presenting the findings to this committee will assist in the validation of the results with the community and provide a basis for reflection and feedback on the relevance of similar interventions in the community. Advisory committee feedback will inform the discussion, recommendations and implication for the practice section of the manuscript.

\section{Patient and public involvement}

This is a scoping review of literature aims to document evidence for the substance use prevention for indigenous elementary school children and is partly informed by project that GM is involved that show early substance use for patients on opioid agonist treatment which showed that clients on this treatment were exposed to polysubstance from 6 to 13 years ${ }^{22}$. Patients and public were not involved in the conceptualisation of the project or the research question.

\section{RESULTS}

The searches, title and abstract screening are ongoing. In the coming months, two independent reviewers will conduct full-text analyses. During the process of fulltext screening, we will also extract data from the marked studies. We expect the scoping review to be completed by late 2019/early 2020.

\section{DISCUSSION}

The decision to focus on indigenous substance use prevention among elementary school children was informed by a project undertaken by GM, on characteristics and predictors of clinical experiences of clients on methadone project. ${ }^{22}{ }^{65}$ In this study, it became clear that the age of exposure to an addictive substance such as opioids, alcohol and marijuana is between 6 and 11 years. Further, most of the clients on opiate replacement therapy struggle with treatment and recovery with more than $70 \%$ dropping out of ORT in the first year. Furthermore, considering that $95 \%$ of the clients on this study self-identified as indigenous, who contend with diverse social determinants of health that are a major barrier to the recovery from addiction, we believe that that emphasis should be placed on understanding the substance use prevention intervention for elementary school children, who are at a risk of substance use.

Understanding interventions to prevent substance use among Indigenous youth aged $7-13$ is vital to addressing substance use and addictions in the Indigenous population. Of interest, we will seek to identify the philosophical and theoretical underpinnings that characterise these interventions and the extent to which Indigenous knowledge is incorporated in these projects. The problem of substance use and addictions cannot be overstated: findings of numerous studies suggest that early exposure to substance use leads to lifelong polysubstance use, which 
not only causes complex comorbid conditions that are difficult and expensive to treat but also severely impacts the quality of life of these individuals and their families. ${ }^{66-68}$ We, therefore, argue that prevention is the best intervention for indigenous communities struggling with substance use challenges, considering they also content with other social determinants of health.

The proposed scoping review has the potential to influence future policy, programmes and services supporting the health and well being of Indigenous youth in Canada and beyond. It will describe the nature and value of early strategies to prevent substance use in this group, and it will provide exemplars of interventions that can be implemented in a Canadian, Australian, New Zealand and US settings.

\section{Limitations of the review}

Since the scoping review aims to assess the research activity on a topic and not the quality of the research thereof, this project will provide an overview of all the intervention focusing on elementary school-based indigenous substance use prevention. The research team recognises that focusing exclusively on substance use prevention projects catering to the 7-13 years old population may limit the number of reviews that can be included in the review. Therefore, some projects which might have provided relevant information to this review might be excluded on account of the age limit. Also, the exclusive focus on school-based interventions, means that any community-based substance use prevention focusing on this age group will be excluded. As a result, the review will not encompass the totality of diverse substance use prevention interventions for this population.

\author{
Author affiliations \\ ${ }^{1}$ College of Nursing, University of Saskatchewan, Prince Albert, Saskatchewan, \\ Canada \\ ${ }^{2}$ University of Saskatchewan College of Nursing, Prince Albert, Saskatchewan, \\ Canada \\ ${ }^{3}$ Library, University of Saskatchewan, Saskatoon, Alberta, Canada \\ ${ }^{4}$ Public Health, University of Saskatchewan College of Graduate Studies and \\ Research, Saskatoon, Saskatchewan, Canada \\ ${ }^{5}$ Nursing, University of Massachusetts Boston, Boston, Massachusetts, USA \\ ${ }^{6}$ Daphne Cockwell School of Nursing, Ryerson University, Toronto, Ontario, Canada \\ ${ }^{7}$ Medicine, University of Saskatchewan College of Medicine, Saskatoon, \\ Saskatchewan, Canada \\ ${ }^{8}$ Nursing, University of Saskatchewan College of Nursing, Saskatoon, \\ Saskatchewan, Canada
}

Contributors GM was involved in the conceptualisation and writing of the protocol. TP was involved in the literature search, writing the introductory section and proofreading the protocol. MM was involved in the literature search, writing the introductory section and proofreading the protocol. MK was involved in designing the search strategy and writing the methods section. AG was involved in editing and proofreading the manuscript. JN was involved in editing and proofreading the manuscript. AK was involved in editing and proofreading the manuscript. SKM was involved in the revision and formatting of the manuscript.

Funding This work was supported by the Faculty Recruitment and Retention Fund, University of Saskatchewan fund no. 419054.

Competing interests None declared.

Patient consent for publication Not required.
Ethics approval Ethics review approval is not required for this project as the project is aimed at providing evidence for the presence of substance use interventions for Indigenous Elementary School children.

Provenance and peer review Not commissioned; externally peer reviewed.

Open access This is an open access article distributed in accordance with the Creative Commons Attribution Non Commercial (CC BY-NC 4.0) license, which permits others to distribute, remix, adapt, build upon this work non-commercially, and license their derivative works on different terms, provided the original work is properly cited, appropriate credit is given, any changes made indicated, and the use is non-commercial. See: http://creativecommons.org/licenses/by-nc/4.0/.

ORCID iD

Geoffrey Maina http://orcid.org/0000-0003-4982-9656

\section{REFERENCES}

1 Gracey M, King M. Indigenous health Part 1: determinants and disease patterns. Lancet 2009;374:65-75.

2 Reid J, Taylor-Moore K, Varona G. Towards a social-structural model for understanding current disparities in Maori health and well-being. Journal of Loss and Trauma 2014;19:514-36.

3 Zambas SI, Wright J. Impact of colonialism on Māori and Aboriginal healthcare access: a discussion paper. Contemp Nurse 2016;52:398-409.

4 Bombay A, Matheson K, Anisman $\mathrm{H}$. The intergenerational effects of Indian residential schools: implications for the concept of historical trauma. Transcult Psychiatry 2014;51:320-38 http://journals. sagepub.com/doi/

5 Hirini PR, Collings SC. Whakamomori, He Whakaaro, He Kōrero Noa Ministry of health 2005.

6 Rege S. State of indigenous mental health in Australia - a colonial legacy? Int Psychiatry 2009;6:98-100.

7 Wirihana R, Smith C. Historical trauma, healing and well-being in Māori communities. He Rau Murimuri Aroha 2019;2.

8 Kirmayer LJ, Brass GM, Tait CL. The mental health of Aboriginal peoples: transformations of identity and community. Can J Psychiatry 2000;45:607-16.

9 Marsh TN, Coholic D, Cote-Meek S, et al. Blending Aboriginal and Western healing methods to treat intergenerational trauma with substance use disorder in Aboriginal peoples who live in northeastern Ontario, Canada. Harm Reduct J 2015;12:14.

10 Gagne MA. The role of dependency and colonialism in generation trauma in First Nations citizens: The James Bay Cree. In: Intergenerational Handbook of multigenerational legacies of trauma. New York: Plenum Press, 1998: 355-71.

11 Ross A, Dion J, Cantinotti M, et al. Impact of residential schooling and of child abuse on substance use problem in Indigenous peoples. Addict Behav 2015;51:184-92 https://linkinghub.elsevier.com/ retrieve/pii/S0306460315002701

12 Nelson S. Challenging hidden assumptions: colonial norms as determinants of Aboriginal mental health. National Collaborating Centre for Aboriginal Health 2013.

13 Mikkonen J, Raphael D. Social determinants of health: the Canadian facts. Toronto: York University School of Health Policy and Management, 2010. //www.thecanadianfacts.org/

14 Reading C, Wien F. Heath inequalities and social determinants of health of Aboriginal people. Prince George BC: National collaborating centre for Aboriginal health, 2013.

15 Gracey M, King M. Indigenous health Part 1: determinants and disease patterns. The Lancet 2009;374:65-75.

16 Kolahdooz F, Nader F, Yi KJ, et al. Understanding the social determinants of health among Indigenous Canadians: priorities for health promotion policies and actions. Glob Health Action 2015;8:27968.

17 King M, Smith A, Gracey M. Indigenous health Part 2: the underlying causes of the health gap. The Lancet 2009;374:76-85.

18 First peoples worldwide. (n.d). The challenges we face. Available: http://www.firstpeoples.org/the-challenges-we-face.htm

19 National collaborating centre for Aboriginal health (n.d.). setting the context: an overview of Aborgiginal health in Canada. Available: https://www.ccnsa-nccah.ca/docs/context/FS-OverviewAbororiginal Health-EN.pdf

20 Urbanoski KA. Need for equity in treatment of substance use among Indigenous people in Canada. Can Med Assoc J 2017;189:E1350-1 http://www.cmaj.ca/lookup/doi/

21 Population health branch $\mathrm{M}$ of $\mathrm{H}$. HIV prevention and control program report for 20152016. 
22 Maina G, Crizzle A, Maposa S, et al. Sociodemographic profiles and clinical outcomes for clients on methadone maintenance treatment in a Western Canadian clinic. J Forensic Nurs 2019.

23 Centre F. First nations regional health survey (Rhs) 2008/10: national report on adults, youth and children living in first nations communities. Ottawa, 2012.

24 Russell C, Firestone M, Kelly L, et al. Prescription opioid prescribing, use/misuse, harms and treatment among Indigenous people in Canada: a narrative review of available data and indicators. Rural Remote Heal 2016;16:3974.

25 Kanate D, Folk D, Cirone S, et al. Community-Wide measures of wellness in a remote first nations community experiencing opioid dependence: evaluating outpatient buprenorphine-naloxone substitution therapy in the context of a first nations healing program. Can Fam Physician 2016:61:160-5.

26 Herring S, Spangaro J, Lauw M, et al. The intersection of trauma, racism, and cultural competence in effective work with Aboriginal people: waiting for trust. Australian Social Work 2013;66:104-17.

27 Durey A. Reducing racism in Aboriginal health care in Australia: where does cultural education fit? Aust N Z J Public Health 2010;34:S87-92.

28 Awofeso N. Racism, a major impediment to optimal Indigenous health and health care in Australia. Australian Indigenous Health Bulletin 2011;11:1-8.

29 Czyzewski K. Colonialism as a broader social determinant of health. Int Indig Policy J 2011;2.

30 Canadian center on substance abuse. substance use and suicide among youth: prevention and intervention strategies, 2016. Available: https://www.ccsa.ca/sites/default/files/2019-04/CCSA-SubstanceUse-Suicide-Prevention-Youth-Summary-2016-en.pdf

31 Aboriginal Healing Foundation. Suicide among Aboriginal people in Canada. Aboriginal healing Foundation. Ottawa, Ontario, 2007. http:// www.ahf.ca/downloads/suicide.pdf

32 Kumar MB, Tjepkema M. Suicide among first nations people, Métis and Inuit (2011-2016): findings from the 2011 Canadian census health and environment cohort (CanCHEC).

33 Canada S. Suicide among First Nations people, Metis and Inuit (2011-2016): Findings from the 2011 Canadian Census Health and Environment Cohort (CanCHEC). [Internet], 2019. Available: https:// www150.statcan.gc.ca/n1/pub/99-011-x/99-011-x2019001-eng.htm

34 Centre for Disease Prevention (CDC). Suicide Risks and Protective Factors. [Internet], 2018. Available: https://www.cdc.gov/ violenceprevention/suicide/riskprotectivefactors.html

35 Kirmayer LJ, Boothroyd LJ, Hodgins S. Attempted suicide among Inuit youth: psychosocial correlates and implications for prevention. Can J Psychiatry 1998;43:816-22.

36 Jetty R, Banerji A, Berry M, et al. Tobacco use and misuse among Indigenous children and youth in Canada. Paediatr Child Health 2017;22:395-9.

37 Statistics Canada. Indigenous Peoples: Fact Sheet for Canada [Internet], 2015. Available: https://www150.statcan.gc.ca/n1/pub/89656-x/89-656-x2015001-eng.htm

38 Moss HB, Chen CM, Yi H-ye. Early adolescent patterns of alcohol, cigarettes, and marijuana polysubstance use and young adult substance use outcomes in a nationally representative sample. Drug Alcohol Depend 2014:136:62.

39 U.S. Department of Health and Human Services (HHS) SA and M, (SAMHSA) HSA. No Title [Internet], 2017. Available: https:// alcoholpolicy.niaaa.nih.gov/sites/default/files/imce/users/u1743/ stop_act_rtc_2017.pdf

40 Swahn MH, Bossarte RM, Ashby JS, et al. Pre-teen alcohol use initiation and suicide attempts among middle and high school students: findings from the 2006 Georgia student health survey. Addict Behav 2010;35:458.

41 King KM, Chassin L. A prospective study of the effects of age of initiation of alcohol and drug use on young adult substance Dependence*. J Stud Alcohol Drugs 2007;68:265.

42 Kirisci L, Tarter R, Ridenour T, et al. Age of alcohol and cannabis use onset mediates the association of transmissible risk in childhood and development of alcohol and cannabis disorders: evidence for common liability. Exp Clin Psychopharmacol 2013;21:45.

43 Hicks JA, Friedman RS, Gable PA, et al. Interactive effects of approach motivational intensity and alcohol cues on the scope of perceptual attention. Addiction 2012;107:1074-80

44 Kennett J, Vincent NA, Snoek A. Drug Addiction and Criminal Responsibility. In: Handbook of Neuroethics [Internet. The Netherlands: Dordrecht: Springer, 2015: 1065-83.
45 Degenhardt L, Randall D, Hall W, et al. Mortality among clients of a state-wide opioid pharmacotherapy program over 20 years: risk factors and lives saved. Drug Alcohol Depend 2009;105:9-15.

46 Sartor CE, Waldron M, Duncan AE, et al. Childhood sexual abuse and early substance use in adolescent girls: the role of familial influences. Addiction 2013;108:993-1000.

47 Chen C-Y, Storr CL, Anthony JC. Early-Onset drug use and risk for drug dependence problems. Addict Behav 2009;34:319-22.

48 Bachman JG, O'Malley PM, Schulenberg JE, et al. Merline AC. The decline of substance use in young adulthood: changes in social activities, roles, and beliefs. Mahwah, NJ, US: Lawrence Erlbaum Associates Publishers, 2002.

49 Hammond CJ, Mayes LC. Potenza Mn. neurobiology of adolescent substance use and addictive behaviors: prevention and treatment implications adolescent medicine. State Arts Rev 2014;25:15.

50 C-YS L, Winters KC, Wall MM. Trajectories of substance use disorders in youth: identifying and predicting group memberships. $J$ Child Adolesc Subst Abuse 2010;19:57.

51 Jordan CJ, Andersen SL. Sensitive periods of substance abuse: early risk for the transition to dependence. Dev Cogn Neurosci 2017;25:44.

52 Caria MP, Faggiano F, Bellocco R, et al. Effects of a school-based prevention program on European adolescents' patterns of alcohol use. J Adolesc Health 2011;48:182-8.

53 Griffin KW, Bang H, Botvin GJ. Age of alcohol and marijuana use onset predicts Weekly substance use and related psychosocial problems during young adulthood. J Subst Use 2010;15:174-83

54 Salvo N, Bennett J, Cheung A, et al. Prevention of of substance use in children/adolescents with mental disorders: can ACAD child Adolesc psychiatry 2012;21:245-52.

55 Logan-Greene P, Semanchin Jones A. Predicting chronic neglect: understanding risk and protective factors for CPS-involved families. Child Fam Soc Work 2018;23:264-72.

56 Goldberg JM, Sklad M, Elfrink TR, et al. Effectiveness of interventions adopting a whole school approach to enhancing social and emotional development: a meta-analysis. Eur J Psychol Educ 2019;34:755-82.

57 Australian Institute of Health and Welfare. Drug use among Aboriginal and Torres Strait Islander peoples. An assessment of data sources, 2006. Available: https://www.aihw.gov.au/getmedia/ a72e5e3b-8316-4242-bed5 e68ad46c6641/duaatsip.pdf.aspx? inline=true

58 Snijder M, Stapinski L, Lees B, et al. Substance use prevention programs for Indigenous adolescents in the United States of America, Canada, Australia and New Zealand: protocol for a systematic review. JMIR Res Protoc 2018;7:e38.

59 Miller TR. How should governments spend the drug prevention dollar: A buyer's guide. In: Stockwell T, Gruenewald P, Toumbourou J, eds. Preventing harmful substance use: the evidence base for policy and practice. England: John Wiley \& Sons, 2005: 415-31.

60 Faggiano F, Vigna-Taglianti F, Burkhart G, et al. The effectiveness of a school-based substance abuse prevention program: 18-month follow-up of the EU-Dap cluster randomized controlled trial. Drug Alcohol Depend 2010;108:56-64.

61 Arksey H, O'Malley L. Scoping studies: towards a methodological framework. Int J Soc Res Methodol 2005;8:19-32.

62 Levac D, Colquhoun H, O'Brien KK. Scoping studies: advancing the methodology. Implementation Sci 2010;5.

63 Ouzzani M, Hammady H, Fedorowicz Z, et al. Rayyan-a web and mobile APP for systematic reviews. Syst Rev 2016;5:210.

64 CADTH. Grey matters: a practical tool for searching health-related grey literature (Internet). Ottawa, 2018. Available: https://www.cadth. $\mathrm{ca} /$ resources/finding-evidence [Accessed 14 Nov 2019].

65 Maina G, Tahir H, Docabo A, et al. Exploring health-care providers' experiences in the care of clients on opioid agonist treatment in two western Canadian clinics. Can J Nurs Res 2019;9:084456211984275.

66 Charles NE, Mathias CW, Acheson A, et al. Preadolescent sensation seeking and early adolescent stress relate to at-risk adolescents substance use by age 15. Addict Behav 2017;69:1-7.

67 De Genna NM, Larkby C, Cornelius MD. Early and adverse experiences with sex and alcohol are associated with adolescent drinking before and during pregnancy. Addict Behav 2007;32:2799-810.

68 Odgers CL, Caspi A, Nagin DS, et al. Is it important to prevent early exposure to drugs and alcohol among adolescents? Psychol Sci 2008; $19: 1037-44$ 